\title{
Long-term urodynamic follow up in pediatric spinal cord injury
}

\author{
R Chao MD, M E Mayo MD \\ University of Washington, Department of Urology, Seattle, Washington, USA
}

Less than $5 \%$ of all spinal cord injuries occur in children under 16 years of age. We have followed up 40 children with spinal cord injury at our medical center. The patients have an average age at presentation of 9.0 years (range newborn to 17 years) and have a mean follow up of 46.1 months (range 1 to 240 months). Twenty-two patients have cervical injuries, 13 thoracic and five have lumbar injuries. Bladder management in this group includes 11 patients with reflex voiding and 29 patients combining anticholinergic medication with intermittent catheterization. Twenty-eight patients have a follow up for more than 1 year. Review of the video urodynamics in this group has shown good function and preservation of the urinary tract in 25 of 28 patients. Treatment failures can be attributed to noncompliance with recommended voiding regimens. Upper tract surveillance using intravenous pyelogram or renal ultrasound has shown preservation of the upper tracts in all patients with anatomically normal lower tracts. We recommend aggressive follow up in this group of patients with yearly renal ultrasound and video urodynamics every 1-2 years to monitor urinary tract function.

Keywords: pediatrics; spinal cord injury; intermittent catheterization; video urodynamics.

\section{Introduction}

The majority of spinal cord injuries occur in the 18 to 25 year old age range. ${ }^{1}$ Estimates for the incidence of pediatric spinal cord injury has ranged from $0.65 \%$ to $9.6 \%$ of all spinal cord injuries. ${ }^{1-3}$ We have followed a significant number of pediatric spinal cord injured patients and will present the method of their long-term urological management and urodynamic follow up. Few studies in the literature have discussed the urological evaluation and management of this population.

\section{Materials and methods}

Forty spinal cord injured children receiving urological care at our institution were identified. These patients either received their

Correspondence: Dr Roberto Chao, Section of Voiding Dysfunction and Urodynamics, Cleveland Clinic FL, 3000 West Cypress Creek Rd, Fort Lauderdale, Florida 33309, USA. initial rehabilitation in our institution or were initially cared for elsewhere and were transferred later for their management. A review of patient charts, video urodynamic studies and radiographic imagings was performed. Patients were classified as reflex voiders if a spontaneous detrusor contraction occurred to empty the bladder at an acceptable voiding pressure. Patients underwent a yearly physical examination, 1-2 year upper tract examinations and video urodynamic evaluation of their urinary tracts. Upper tract examinations included either intravenous pyelograms or renal ultrasound.

The patient population consisted of 40 spinal cord injured children less than 18 years of age. Ten patients had complete lesions, 14 incomplete and 16 unknown. The majority of spinal cord injured patients had had motor vehicle accidents: 13 as pedestrians and 11 as passengers. Four patients were injured at birth during delivery. Four suffered gun shot wounds. Three patients had episodes of transverse myelitis. A small 
number of patients were involved in accidents with motorcycles, diving, falls and boats.

\section{Results}

Of the population, 22 patients had cervical cord injuries (15 male and seven female). Thirteen patients had thoracic cord injuries (11 male and two female), and five patients lumbar injuries (three male and two females).

Overall, the patients have an average age at presentation of 9.0 years (range newborn to 17 years), with a mean follow-up time of 46.1 months (range 1 to 240 months). Individual follow up and ages at presentation are summarized in Table I.

Reflex voiding or catheterization was recommended depending on the patient's bladder voiding pressure, on urodynamics, the child's age, and the family situation. Those patients having moderate to severe trabeculation and correspondingly high pressures (detrusor $>40 \mathrm{cmH}_{2} \mathrm{O}$ ), or any patient exhibiting detrusor-sphincter dysynergia on video urodynamics were placed on anticholinergic drugs and intermittent catheterization (ICP). Patients and families desiring continence also started ICP, with medications if indicated. In the cervical injury group, the average age at presentation was 7.8 years, with a mean follow up of 56.7 months. Urodynamic studies, performed every 1-2 years, showed that all patients had a hyperreflexic bladder. Spinal injured infants had an increase in bladder capacity as they grew; older individuals had a functional reduction in their capacity. The average cystographic appearance of patient's bladders at the last urodynamic fol- low up was smooth to slightly trabeculated. Eight patients (including one vesicostomy) are reflex voiders. Fourteen patients are performing intermittent catheterization and are on anticholinergic drugs.

Patients with thoracic injuries presented at an average age of 9.4 years, with a mean follow up of 52.9 months. Urodynamic studies showed that 11 patients had a hyperreflexic bladder and two were arreflexic. Bladder management, based on the detrusor pressures generated, the cystographic bladder appearance, and the social situation of the patient allowed two patients to be reflex voiders, and 11 are on ICP/anticholinergics.

Patients with lumbar injuries presented at an average age of 9.8 years, with a mean follow up of 28.8 months. Urodynamic studies showed that all of these patients had arreflexic bladders. Two patients are voiding with Valsalva's manoeuver and three patients are on ICP/anticholinergic drugs.

\section{Long-term follow up}

Twenty-eight patients from this population were followed for more than 1 year (Table II). Video fluoroscopy during urodynamic studies at the last follow up showed the bladder wall to be smooth in $43 \%(12 / 28)$, mildly trabeculated in $50 \%(14 / 28)$, and markedly trabeculated in $7 \%(2 / 28)$. Five patients had a change from a smooth bladder wall to mild trabeculation (four cervical injuries and one thoracic injury). Four patients had a change from a trabeculated bladder wall to a smooth wall (three cervical injuries and one thoracic injury) on six month follow up after institution of ICP and anticholinergic therapy. One patient had a

Table I Results

\begin{tabular}{lccc} 
& Cervical SCI & Thoracic SCI & Lumbar SCI \\
Patients & 22 & 13 & 5 \\
Male/female & $15 / 7$ & $11 / 2$ & $3 / 2$ \\
\hline Average age & 7.8 years & 9.4 years & 9.8 years \\
Follow up & 56.7 months & 52.9 months & 28.8 months \\
Voiding & & & 2 (Valsalva) \\
$\quad$ Reflex & $8(1$ vesicostomy) & 2 & 3 \\
ICP/Meds & 14 & 11 & \\
\hline
\end{tabular}


Table II Long-term results

\begin{tabular}{lccc}
\hline & Cervical SCI & Thoracic SCI & Lumbar SCI \\
Patients & 19 & 6 & 3 \\
\hline Average age & 5.5 years & 5.5 years & 11 years \\
Follow up & 69.2 months & 118 months & 44 months \\
Voiding & & & 2 (Valsalva) \\
$\quad$ Reflex & $8(1$ vesicostomy) & 1 & 1 \\
ICP/Meds & 11 & 5 & \\
\hline
\end{tabular}

grade $2 / 5$ vesicoureteral reflux, which resolved on ICP/anticholinergics. No other significant upper tract changes occurred during the follow up of the patients.

In this group, 11 patients are reflex voiders: five patients had smooth bladders on cystography, four with mild trabeculation and two with moderate or severe trabeculation at last follow up. The latter two patients were recommended to start ICP/anticholinergics, but have not complied. Seventeen patients are on ICP/ anticholinergics: nine had smooth bladders, eight with mild trabeculation and more with severely trabeculated bladders. Of the nine reflex voiders with smooth to mildly trabeculated bladders, six are cervical injuries (complete and incomplete lesions), one thoracic (congenital) and two lumbar (one complete and one incomplete).

\section{Discussion}

Approximately seven to 8,000 spinal cord injuries occur in the United States per year. ${ }^{3}$ Those under 15 years of age comprise only $1-3 \%$ of injuries. Thus children and adolescents are rarely involved. The pediatric spine is more predisposed to certain fractures because of its increased physiological mobility secondary to ligamentous laxity, incomplete ossification of facet joints and vertebral bodies, and underdeveloped neck musculature. In children, the greatest cervical motion occurs at the C2-3 level. By 8-10 years of age, this shifts downward to the C5-6 adult level. ${ }^{4}$

The most common cause of spinal cord injury in all age groups is trauma. For children, motor vehicle accidents $(41.5 \%)$ are the leading etiology followed by sports
$(27 \%)$, violence $(20.5 \%)$ and falls $(9.4 \%))^{2,3}$ We saw a similar etiology of injury in our population. Once injured, the majority of the patients have hyperreflexic bladders. There was a tendency in our long-term study group to have a higher number of reflex voiders.

There are only a few reported series in the literature of pediatric spinal cord injured patients. In 1974, Burke $^{7}$ documented 29 traumatically paralyzed children. Follow up was by monitoring of IVP, urinalysis and serum creatinine. Of 26 evaluable patients, 20 were injured less than 10 years. Of these short-term patients, 17 had normal upper tracts. Voiding in this group included 15 balanced bladders, four with indwelling catheters and one with a right nephrostomy. Six patients were injured for 10 years or more. Only one of these six patients had a normal IVP. Voiding was catheter free in one, four had catheters and one had bilateral cutaneous ureterostomies. Fanciullacci et $a l^{8}$ reported on 18 children with spinal cord injury. On initial urological evaluation ( 2 months to 1.5 years from injury), 13 of 18 patients had normal upper tracts. Initial urodynamic data showed 12 hyperreflexic bladders (six with detrusorsphincter dysynergia), three arreflexic bladders and three unknowns. Upper tract follow up in 14 patients (by IVP or renal scan) with conservative management at a mean of 7.7 years from injury showed normal upper tracts in eight of 14 patients, only one being managed with $\mathrm{ICP} /$ anticholinergics. Therefore, six of 14 patients $(43 \%)$ showed deterioration of their upper urinary tracts. Both series show that without appropriate urodynamic monitoring of detrusor pressures, patients run the risk of long-term damage to their upper tracts. 
Video urodynamics and radiographic studies of the urinary tract in our group were helpful in identifying those patients with high-pressure bladders and detrusorsphincter dysynergia and at risk of upper tract damage. Those patients voiding on their own and showing increasing detrusor trabeculation or reflux with high-pressure voiding were placed on an ICP/anticholinergic regimen.

Urinary tract management goals after spinal cord injury include bladder emptying, continence, infection prevention and preservation of the upper tracts. ${ }^{3}$ In those patients followed for 1 year or longer, treatment failures could be attributed to noncompliance with recommended voiding regimens. It is interesting to see the tendency to reflex voiding in the pediatric population. Of the 28 patients with long-term hyperreflexic bladders, seven $(25 \%)$ are able to reflexively void. It is definitely rare to see an adult male SCI patient who is a reflex voider without the aid of sphincterotomy. Preservation of the urinary tract has been accomplished in those patients to date with reflex voiding patterns.

To summarize, 39 pediatric spinal cord injured patients and their follow-up urodynamics are presented. Twenty-eight of these patients have been followed up for more than 1 year. Intermittent catheterization and anticholinergic drugs were recommended to attain continence or if bladder wall changes occurred. Thus, 11 patients voided reflexively (including one with vesicostomy) and 28 patients were on intermittent catheterization and anticholinergics. Twenty-six out of 28 patients following recommended voiding protocols have maintained their urinary tracts.

We may conclude from this study that pediatric as compared with adult spinal cord injury patients have a higher prevalence of reflex voiding with more 'balanced' bladders. Reflex voiding is safe if regular and careful follow up can be guaranteed so that intermittent catheterization and anticholinergics can be started if bladder wall changes occur. Although intermittent catheterization has been advocated for all children with bladder neuropathy after spinal cord injury, ${ }^{3}$ in younger children it is not necessary for social reasons and may add an extra burden for the family. In older tetraplegic patients, reflex voiding rather than intermittent catheterization will allow more independence from their parents or caregivers, but some of the males may require sphincterotomy as they approach adulthood. To date, no augmentations have been performed which are in contrast to the experience in children with myelodysplasia. Periodic urinary tract assessment with renal ultrasound or intravenous pyelogram and video urodynamics is essential to monitor urinary tract morphology and renal function.

\section{References}

1 Massagli TL, Jaffe KM (1990) Pediatric spinal cord injury: treatment and outcome. Pediatrician 17: 244-254.

2 Kervalramani LS, Kraus JF, Sterling HM (1980) Acute spinal-cord lesions in a pediatric population: epidemiological and clinical features. Paraplegia 18: 206-219.

3 Dector RM, Bauer SB (1993) Urologic management of spinal cord injury in children. Urologic Clin N Am 20: $475-483$.

4 McGuire EJ, Woodside JR, Bordon TA et al (1981) Prognostic value of urodynamic testing in myelodysplasic patients. J Urology 126: 205-208.

5 Wilberger JE (1986) Spinal Cord Injuries in Children. New York, Futura Publishing, vii-xi.

6 Jones ET, Hensinger RN (1982) Cervical spine injuries in children. Contemp Orthop 5: 17-23.

7 Burke DC (1974) Traumatic spinal paralysis in children. Paraplegia 11: 268-276.

8 Fanciullacci F, Zanollo A, Sandri S, Catazaro F (1988) The neuropathic bladder in children with spinal cord injury. Paraplegia 26: 83-86. 